\title{
Clustering Distributed Sensor Data Streams
}

\author{
Pedro Pereira Rodrigues ${ }^{1,2}$, João Gama ${ }^{1,3}$, and Luís Lopes ${ }^{2,4}$ \\ ${ }^{1}$ LIAAD - INESC Porto L.A. \\ Rua de Ceuta, 118 - 6 andar, 4050-190 Porto, Portugal \\ ${ }^{2}$ Faculty of Sciences of the University of Porto, Portugal \\ pprodrigues@fc.up.pt \\ ${ }^{3}$ Faculty of Economics of the University of Porto, Portugal \\ jgama@fep.up.pt \\ ${ }^{4}$ CRACS - INESC Porto L.A. \\ lblopes@dcc.fc.up.pt
}

\begin{abstract}
Nowadays applications produce infinite streams of data distributed across wide sensor networks. In this work we study the problem of continuously maintain a cluster structure over the data points generated by the entire network. Usual techniques operate by forwarding and concentrating the entire data in a central server, processing it as a multivariate stream. In this paper, we propose DGClust, a new distributed algorithm which reduces both the dimensionality and the communication burdens, by allowing each local sensor to keep an online discretization of its data stream, which operates with constant update time and (almost) fixed space. Each new data point triggers a cell in this univariate grid, reflecting the current state of the data stream at the local site. Whenever a local site changes its state, it notifies the central server about the new state it is in. This way, at each point in time, the central site has the global multivariate state of the entire network. To avoid monitoring all possible states, which is exponential in the number of sensors, the central site keeps a small list of counters of the most frequent global states. Finally, a simple adaptive partitional clustering algorithm is applied to the frequent states central points in order to provide an anytime definition of the clusters centers. The approach is evaluated in the context of distributed sensor networks, presenting both empirical and theoretical evidence of its advantages.
\end{abstract}

Keywords: online adaptive clustering, distributed data streams, sensor networks, incremental discretization, frequent items monitoring.

\section{Introduction}

Data gathering and analysis have become ubiquitous, in the sense that our world is evolving into a setting where all devices, as small as they may be, will be able to include sensing and processing ability. Nowadays applications produce infinite streams of data distributed across wide sensor networks. Therefore, data should also be processed by sensor networks in a distributed fashion. Usual techniques operate by forwarding and concentrating the entire data in a central server, 
processing it as a multivariate stream. In this work we study the problem of continuously maintain a cluster structure over the data points generated by the entire network, where each sensor produces a univariate stream of data. The main problem in applying clustering to data streams is that systems should consider data evolution, being able to compress old information and adapt to new concepts. In this paper, we propose a new distributed algorithm which reduces both the dimensionality and the communication burdens.

In the next section we present some related work on the research areas addressed by this paper. In section 3 our method is explained, with relevant analysis of the overall processing. Section 4 focus on the advantages presented by our proposal in terms of memory and communication resources, especially important in distributed sensor networks. Validation of the system and experimental results on real-world scenarios are presented in section 5. Final section concludes the paper, with thorough discussion and conclusions, including foreseen future work.

\section{Related Work}

The method we present in this paper is related with three other areas of research in data streams: sensor network processing, frequent items monitoring, and distributed clustering.

\subsection{Sensor Data and Networks}

Sensors are usually small, low-cost devices capable of sensing some attribute of a physical phenomenon. In terms of hardware development, the state-of-the-art is well represented by a class of multi-purpose sensor nodes called motes [8], which were originally developed at UC Berkeley and are being deployed and tested by several research groups and start-up companies. Although common applications are traditionally developed on low-level programming of the motes, recent programming languages and environments such as Regiment 21] and EnviroSuite [18, provide high level programming abstractions, allowing more complex programming and usage of sensor devices as processing units for knowledge extraction scenarios. Sensor networks are composed of a variable number of sensors (depending on the application), which have several distinguishing features: (a) the number of nodes is potentially very large and thus scalability is a problem, (b) the individual sensors are prone to failure given the often challenging conditions they experiment in the field, (c) the network topology changes dynamically, (d) broadcast protocols are used to route messages in the network, (e) limited power, computational, and memory capacity, and (f) lack of global identifiers [3]. In sensor network applications, data routing is usually based on two main approaches: (a) sensors broadcast advertisements for the availability of the data and wait for interested nodes, or; (b) sinks broadcast interest in the data and wait for replies from the sensors. Common problems with these strategies are implosion and overlap [3]. 


\subsection{Frequent Items in Data Streams}

The problem of finding the most frequent items in a data stream $S$ of size $N$ is, roughly put, the problem to find the elements $e_{i}$ whose relative frequency $f_{i}$ is higher than a user specified support $\phi N$, with $0 \leq \phi \leq 1$. Given the space requirements that exact algorithms addressing this problem would need [5], several algorithms were already proposed to find the top- $k$ frequent elements, being roughly classified into counter-based and sketch-based [20. Counter-based techniques keep counters for each individual element in the monitored set, which is usually a lot smaller than the entire set of elements. When an element is seen which is not currently being monitored, different algorithms take different actions in order to adapt the monitored set accordingly. Sketch-based techniques provide less rigid guarantees, but they do not monitor a subset of elements, providing frequency estimators for the entire set.

Simple counter-based algorithms such as Sticky Sampling and Lossy Counting were proposed in [19], which process the stream in reduced size. Yet, they suffer from keeping a lot of irrelevant counters. Frequent [10] keeps only $k$ counters for monitoring $k$ elements, incrementing each element counter when it is observed, and decrementing all counters when a unmonitored element is observed. Zeroedcounted elements are replaced by new unmonitored element. This strategy is similar to the one applied by Space-Saving [20, which gives guarantees for the top- $m$ most frequent elements. Sketch-based algorithms usually focus on families of hash functions which project the counters into a new space, keeping frequency estimators for all elements. The guarantees are less strict but all elements are monitored. The CountSketch algorithm [5] solves the problem with a given success probability, estimating the frequency of the element by finding the median of its representative counters, which implies sorting the counters. Also, Group Test method [6] employs expensive probabilistic calculations to keep the majority elements within a given probability of error. Although generally accurate, its space requirements are large and no information is given about frequencies or ranking.

\subsection{Clustering Data Streams}

The main problem in applying clustering to data streams is that systems should consider data evolution, being able to compress old information and adapt to new concepts. The range of clustering algorithms that operate online over data streams is wide, including partitional, hierarchical, density-based and grid-based methods. A common connecting feature is the definition of unit cells or representative points, from which clustering can be obtained with less computational costs. In this paper we address two types of clustering procedures: point-based clustering and grid-based clustering.

Point-Based Clustering. Several algorithms operate over summaries or samples of the original stream. Bradley et al. 4] proposed the Single Pass K-Means, increasing the capabilities of $k$-means for large datasets, by using a buffer where points of the dataset are kept in a compressed way. The STREAM 22] system 
can be seen as an extension of [4] which keeps the same goal but has as restriction the use of available memory. After filling the buffer, STREAM clusters the buffer into $k$ clusters, retaining only the $k$ centroids weighted by the number of examples in each cluster. The process is iteratively repeated with new points. The BIRCH hierarchical method [25] uses Clustering Features to keep sufficient statistics for each cluster at the nodes of a balanced tree, the $C F$-tree. Given its hierarchical structure, each non-leaf node in the tree aggregates the information gathered in the descendant nodes. This algorithm tries to find the best groups with respect to the available memory, while minimizing the amount of input and output. Another use of the $C F$-tree appears in 1 . A different strategy is used in another hierarchical method, the CURE system [17, where each cluster is represented by a constant number of points well distributed within the cluster, which capture the extension and shape of the cluster. This process allows the identification of clusters with arbitrary shapes on a random sample of the dataset, using Chernoff bounds in order to obtain the minimum number of required examples. The same principle of error-bounded results was recently used in VFKM to apply consecutive runs of k-means, with increasing number of examples, until the error bounds were satisfied [1]. This strategy supports itself on the idea of guaranteeing that the clustering definition does not differ significantly from the one gather with infinite data. Hence, it does not consider data evolution.

Grid-Based Clustering. The main focus of grid-based algorithms is the so called spatial data, which model the geometric structure of objects in space. These algorithms divide the data space in small units, defining a grid, and assigning each object to one of those units, proceeding to divisive and aggregative operations hierarchically. These features make this type of methods similar to hierarchical algorithms, with the main difference of applying operations based on a parameter rather than the dissimilarities between objects. A sophisticated example of this type of algorithms is STING [24, where the space area is divided in cells with different levels of resolution, creating a layered structure. The main features and advantages of this algorithm include being incremental and able of parallel execution. Also, the idea of dense units, usually present in density-based methods [12, has been successfully introduced in grid-based systems. The CLIQUE algorithm tries to identify sub-spaces of a large dimensional space which can allow a better clustering of the original data 2]. It divides each dimension on the same number of equally ranged intervals, resulting in exclusive units. One unit is accepted as dense if the fraction of the total number of points within the unit is higher than a parameter value. A cluster is the largest set of contiguous dense units within a subspace. This technique's main advantage is the fact that it automatically finds subspaces of maximum dimensionality in a way that high density clusters exist in those subspaces. The inclusion of the notion of dense units in simpler grid-based methods presents several benefits. However, in distributed systems, the increase in communication given the need to keep sufficient statistics may be prejudicial. 
Distributed Clustering. Since current applications generate many pervasive distributed computing environments, data mining systems must nowadays be designed to work not as a monolithic centralized application but as a distributed collaborative process. The centralization of information yields problems not only with resources such as communication and memory, but also with privacy of sensitive information. Instead of centralizing relevant data in a single server and afterwards perform the data mining operations, the entire process should be distributed and, therefore, paralleled throughout the entire network of processing units. A recent example of such techniques was proposed by Subramaniam et al., where an online system uses density distribution estimation to detect outliers in distributed sensor data 23. Recent research developments in clustering are directed towards distributed algorithms for continuous clustering of examples over distributed data streams. In [9] the authors present a distributed majority vote algorithm which can be seen as a primitive to monitor a k-means clustering over peer-to-peer networks. The k-means monitoring algorithm has two major parts: monitoring the data distribution in order to trigger a new run of k-means algorithm and computing the centroids actually using the k-means algorithm. The monitoring part is carried out by an exact local algorithm, while the centroid computation is carried out by a centralization approach. The local algorithm raises an alert if the centroids need to be updated. At this point data is centralized, a new run of k-means is executed, and the new centroids are shipped back to all peers. Cormode et al. 7] proposed different strategies to achieve the same goal, with local and global computations, in order to balance the communication costs. They considered techniques based on the furthest point algorithm [16], which gives a approximation for the radius and diameter of clusters with guaranteed cost of two times the cost of the optimal clustering. They also present the parallel guessing strategy, which gives a slightly worse approximation but requires only a single pass over the data. They conclude that, in actual distributed settings, it is frequently preferable to track each site locally and combine the results at the coordinator site. These methods of combining local and central processing are paradigmatic examples of the path that distributed data mining algorithms should traverse.

\section{$3 \quad$ DGClust - Distributed Grid Clustering}

In this section we present DGClust, a distributed grid clustering system for sensor data streams. Each local sensor receives data from a given source, producing a univariate data stream, which is potentially infinite. Therefore, each sensor's data is processed locally, being incrementally discretized into a univariate adaptive grid. Each new data point triggers a cell in this grid, reflecting the current state of the data stream at the local site. Whenever a local site changes its state, that is, the triggered cell changes, the new state is communicated to a central site. Furthermore, the central site keeps the global state of the entire network where each local site's state is the cell number of each local site's grid. Nowadays, sensor networks may include thousands of sensors. This scenario yields an exponential 
number of cell combinations to be monitored by the central site. However, it is expected that only a small number of this combinations are frequently triggered by the whole network, so, parallel to the aggregation, the central site keeps a small list of counters of the most frequent global states. Finally, the current clustering definition is defined and maintained by a simple adaptive partitional clustering algorithm applied on the frequent states central points.

\subsection{Notation and Formal Setup}

The goal is to find $k$ cluster centers of the data produced by a network of $d$ local sensors. Let $X=\left\{X_{1}, X_{2}, \ldots, X_{d}\right\}$ be the set of $d$ univariate data streams, each of which is produced by one sensor in one local site. Each local site $i$ keeps a twolayered discretization of the univariate stream, with $p_{i}$ intervals in the first layer and $w_{i}$ intervals in the second layer, where $k<w_{i}<<p_{i}$ but $w_{i} \in O(k)$. At each time $t$, each local sensor produces a value $X_{i}(t)$ and defines its local discretized state $s_{i}(t)$, drawn from the set of possible states $S_{i}$, the unit cells in the univariate grid $\left(\left|S_{i}\right|=w_{i}\right)$. If no value is read, or $s_{i}(t)=s_{i}(t-1)$, no information is sent to the central site. The central site monitors the global state of the network at time $t$, by combining each local discretized state $s(t)=\left\langle s_{1}(t), s_{2}(t), \ldots, s_{d}(t)\right\rangle$. Each $s(t)$ is drawn from a finite set of cell combinations $E=\left\{e_{1}, e_{2}, \ldots, e_{|E|}\right\}$,

with $|E|=\prod_{i=1}^{d} w_{i}$. Given the exponential size of $E$, the central site monitors only a subset $F$ of the top- $m$ most frequent elements of $E$, with $k<|F|<<|E|$. Relevant focus is given to size requirements, as $|E| \in O\left(k^{d}\right)$ but $|F| \in O\left(d k^{\beta}\right)$, with small $\beta$. Finally, the top- $m$ frequent states central points are used in an online adaptive partitional clustering algorithm, which defines the current $k$ cluster centers, being afterwards continuously adapted.

\subsection{Local Adaptive Grid}

Discretization of continuous attributes is an important task for certain types of machine learning algorithms. Although discretization is a well-known topic in data analysis and machine learning, most of the works refer to a batch discretization where all the examples are available for discretization. Few works refer to incremental discretization. However, it is commonly seen as an essential tool for high-speed processing of data streams with limited memory resources [14. For example, grid clustering algorithms operate on discrete cells to define dense regions of points [24]. In our approach, the main reason to perform discretization of each univariate data stream in sensor networks is to considerably reduce the communication with the central server, which tries to capture the most frequent regions of the entire input space.

Partitional Incremental Discretization. In our approach, we apply incremental discretization at each sensor univariate stream $X_{i}$ using the Partition Incremental Discretization (PiD) algorithm [13], which is composed by two layers. The first layer simplifies and summarizes the data, while the second layer constructs the final grid. For the scope of this work, we only consider equal-width 
discretization. The first layer is initialized based on the number of intervals $p_{i}$ (that should be much larger than the desired final number of intervals $w_{i}$ ) and the range of the variable. The range of the variable is only indicative, as it is used to define the intervals in the first layer using a equal-width strategy, but it is not rigid as layer-one bins can be split if necessary, monitoring the actual range of the values. Each time a new value $X_{i}(t)$ is read by the sensor $i$, we increment the counter in the corresponding interval. After a given number of examples, the second layer can be defined. The process of updating the first layer works online, doing a single scan over the data stream, hence being able to process infinite sequences of data, processing each example in constant time and (almost) constant space. We consider a split operator to be triggered in a given layer-one bin as soon as the number of hits in it is above a user-defined threshold $\alpha$ (a percentage of the total number of points considered so far). This split operator generates new intervals in layer one, adapting the initial intervals with the actual distribution of values. This is especially important to correct badly initialized range values and to adapt to slight changes in the distribution of values. The second layer merges the set of intervals defined by the first layer, and defines the final univariate discretization grid $\left\langle b_{1}, b_{2}, \ldots, b_{w_{i}}\right\rangle$, with size $w_{i}>k$ linear to the number of final clusters to find. The grid this way defined represents an approximated histogram of the variable produced at the local site. The update process of the second layer works online along with the first layer. For each new example $X_{i}(t)$, the system increments the counter in the second-layer cell where the triggered first-layer cell is included, defining the discretized state $s_{i}(t)$. Algorithm 1 presents the adaptive discretization procedure executed at each local site.

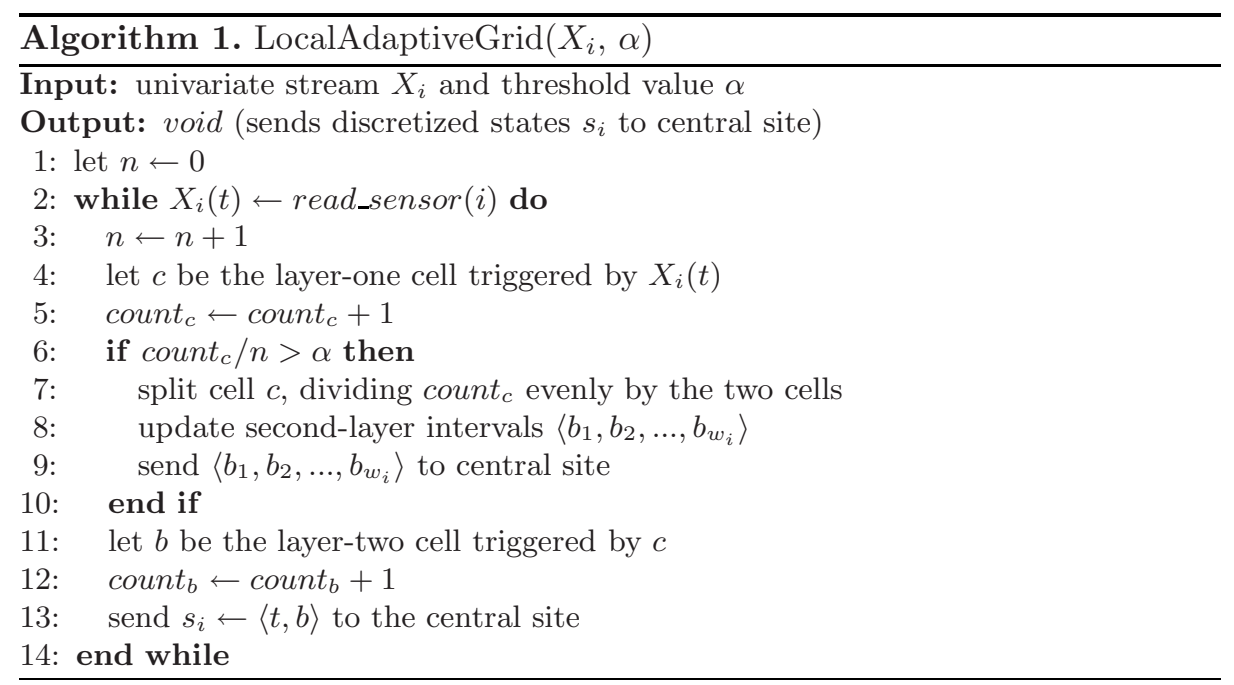




\subsection{Centralized Frequent State Monitoring}

In this work we consider synchronous processing of sensor data. The global state is updated at each time stamp as a combination of each local site's state, where each value is the cell number of each local site's grid, $s(t)=\left\langle s_{1}(t), s_{2}(t), \ldots, s_{d}(t)\right\rangle$. If in that period no information arrives from a given local site $i$, the central site assumes that site $i$ stays in the previous local state $\left(s_{i}(t) \leftarrow s_{i}(t-1)\right)$. The number $|E|$ of cell combinations to be monitored by the central site is exponential to the number of sensors, $|E|=O\left(w^{d}\right)$. However, only a small number of this combinations represent states which are frequently visited by the whole network. This way the central site keeps a small list, $F$, of counters of the most frequent global states, whose central points will afterwards be used in the final clustering algorithm, with $|F|=O\left(d k^{\beta}\right)$, for small $\beta$.

Space-Saving Top- $\boldsymbol{m}$ Elements Monitoring. Each seen global state $e \in E$ is a frequent element $f_{r}$ whose counter count $t_{r}$ currently estimates that it is the $r^{t h}$ most frequent state. The system applies the Space-Saving algorithm [20, to monitor only the top- $m$ elements. If we observe a state $s(t)$ that is monitored in $\operatorname{rank} r\left(f_{r}=s(t)\right)$, we just increment its counter, count $t_{r}$. If $s(t)$ is not monitored, replace $f_{m}$, the element that currently has the least estimated hits, count $t_{m}$, with $s(t)$, and increment count $_{m}$. For each monitored element $f_{i}$, we keep track of its over-estimation, $\varepsilon_{i}$, resulting from the initialization of its counter when it was inserted into the list. That is, when starting to monitor $f_{i}$, set $\varepsilon_{i}$ to the value of the evicted counter. An element $f_{i}$ is guaranteed to be among the top- $m$ elements if its guaranteed number of hits, count ${ }_{i}-\varepsilon_{i}$, exceeds count $_{m+1}$. The authors report that, even if it is not possible to guarantee top- $m$ elements, the algorithm can guarantee top- $m^{\prime}$ elements, with $m^{\prime} \approx m$. Hence, suitable values for $m$ should be considered. Furthermore, due to errors in estimating the frequencies of the elements, the order of the elements in the data structure might not reflect their exact ranks. Thus, when performing clustering on the top- $m$ elements, we should be careful not to directly weight each point by its rank. The goal of this strategy is to monitor top- $m$ states, using only the guaranteed top- $m$ elements as points for the final clustering algorithm. One important characteristic of this algorithm is that it tends to give more importance to recent examples, enhancing the adaptation of the system to data evolution.

\subsection{Centralized Online Clustering}

The goal of DGClust is to find and continuously keep a cluster definition, reporting the $k$ cluster centers. Each frequent state $f_{i}$ represents a multivariate point, defined by the central points of the corresponding unit cells $s_{i}$ for each local site $X_{i}$. As soon as the central site has a top- $m$ set of states, with $m>k$, a simple partitional algorithm can be applied.

Initial Centers. In the general task of finding $k$ centers given $m$ points, there are two major objectives: minimize the radius (maximum distance between a 
point and its closest cluster center) or minimize the diameter (maximum distance between two points assigned to the same cluster) 7. The Furthest Point algorithm [16] gives a guaranteed 2-approximation for both the radius and $d i$ ameter measures. It begins by picking an arbitrary point as the first center, $c_{1}$, then finding the remaining centers $c_{i}$ iteratively as the point that maximizes its distance from the previously chosen centers $\left\{c_{1}, \ldots, c_{i-1}\right\}$. After $k$ iterations, one can show that the chosen centers $\left\{c_{1}, c_{2}, \ldots, c_{k}\right\}$ represent a factor 2 approximation to the optimal clustering [16]. See 7] for a proof. This strategy gives a good initialization of the cluster centers, computed by finding the center $k_{i}$ of each cluster after attracting remaining points to the closest center $c_{i}$. This algorithm is applied as soon as the system finds a set of $m^{\prime}>k$ guaranteed top- $m$ states.

Continuous Adaptive Clustering. It is known that a single iteration is not enough to converge to the actual centers in simple $k$-means strategies. Hence we consider two different states on the overall system operation: converged and non-converged. At every new state $s(t)$ that is gathered by the central site, if the system has not yet converged, it adapts the clusters centers using the $m^{\prime}$ guaranteed top- $m$ states. On the other hand, if the system has converged, two different scenarios may occur. If the current state is being monitored as one of the $m^{\prime}$ top- $m$ states, then the set of points actually used in the final clustering is the same, so the clustering centers remain the same. No update is performed. On the other hand, if the current state has just become guaranteed top- $m$, then the clusters may have change so we move into a non-converged state of the system, updating the cluster centers. Another scenario where the clusters centers require adaptation is when one or more local sites transmit their new grid intervals, which are used to define the central points of each state. In this case we also update and move to non-converged state. A different scenario is created when a new state enters the top- $m$, replacing the least frequent one. In this case, some of the previously guaranteed top- $m$ may loose their guarantee. However, if the list of frequent items is small (imposed by resources restrictions) this will happen very frequently so we disregard this scenarios to prevent excessive computation when cluster centers have already converged. Future work will focus on these scenarios for concept drift and cluster evolution purposes. In scenarios where clusters centers adaptation is needed, our system updates the clustering definition by applying a single iteration of point-to-cluster assignment and cluster centers computation. This process assures a smooth evolution of the cluster centers, while it nevertheless adapts them to the most recent data, as old data points tend to be less frequent. Algorithm 2 presents the central adaptive procedure executed at the server site. The algorithm for ClusterCentersUpdate $(K, F)$ is omitted for simplicity and space saving. Figure 1 presents a final grid, frequent cells and cluster centers for a specific case with $d=2, k=5$, for different values of $w$ and $m$. The flexibility of the system is exposed, as different parameter values yield different levels of results. Moreover, the continuous update keeps track of the most frequent cells keeping the gathered centers within acceptable bounds. A good characteristic of the system is this ability to adapt to resource restricted 


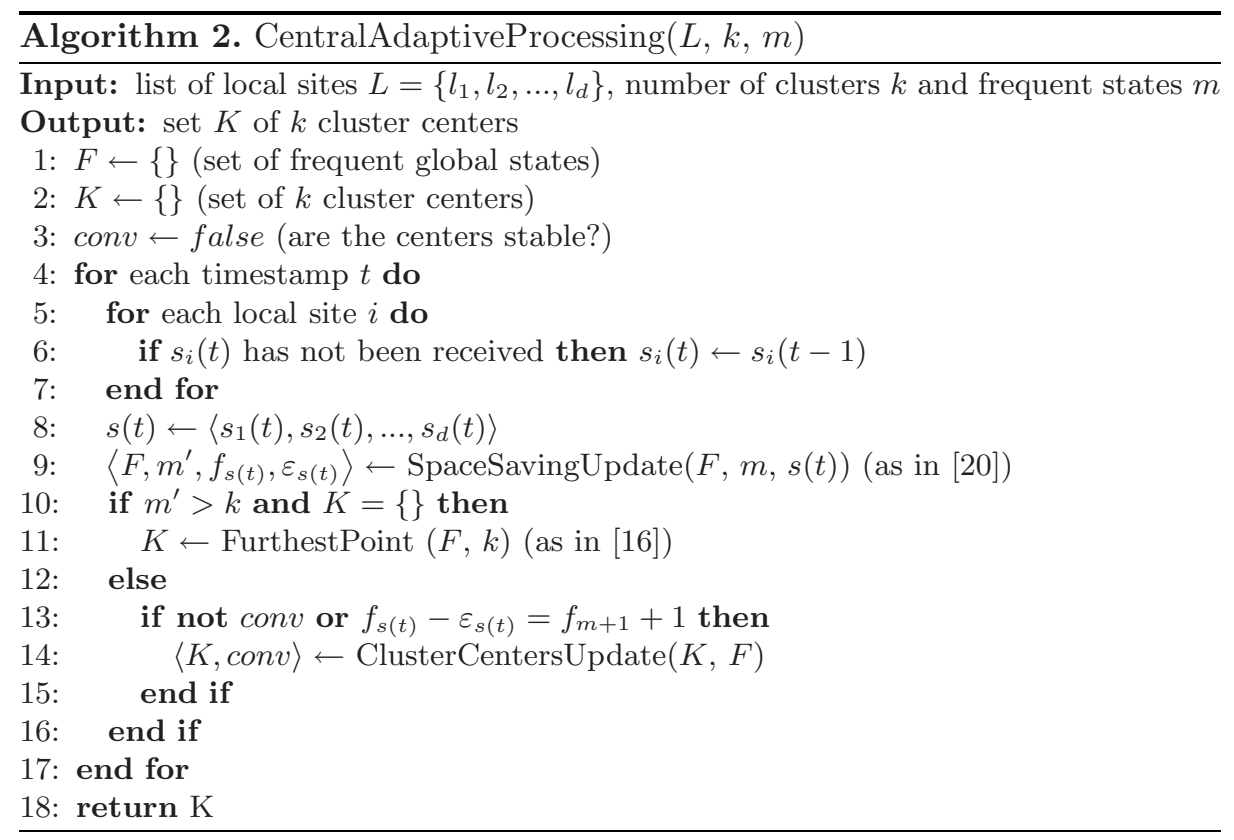

environments: system granularity can be defined given the resources available in the network's processing sites.

\section{Algorithm Analysis}

Each univariate data stream is discretized, with only the discretized state being forwarded to the central site. At this point, the granularity of each sensor's grid will directly influence the error in that dimension. Since the construction of the second layer is directly restricted to the intervals defined in layer one, the final histograms will also be an approximation of the exact histograms that would be defined directly if all data was considered. Nevertheless, with this two-layer strategy the update of the final grid is straightforward. The layer-two intervals just need to be recomputed when the split operator in layer one is triggered. Moreover, the number of intervals in the second layer can be adjusted individually for each sensor, in order to address different needs of data granularity and resources requirements. In this proposal we address univariate sensor readings. The data stream model we consider in sensor networks assumes that a sensor value represents its state in a given moment in time. If the readings of a local sensor fall consecutively in the same layer-two interval, no sound information would be given to the central site. Thus, local sites only centralize information when a new value triggers an interval different from the previously sent to the central server. The central site only monitors the top- $m$ most frequent global states, disregarding infrequent states which could influence the final clusters. Finally, the system performs partitional clustering over the $m^{\prime}$ guaranteed top- $m$ frequent 

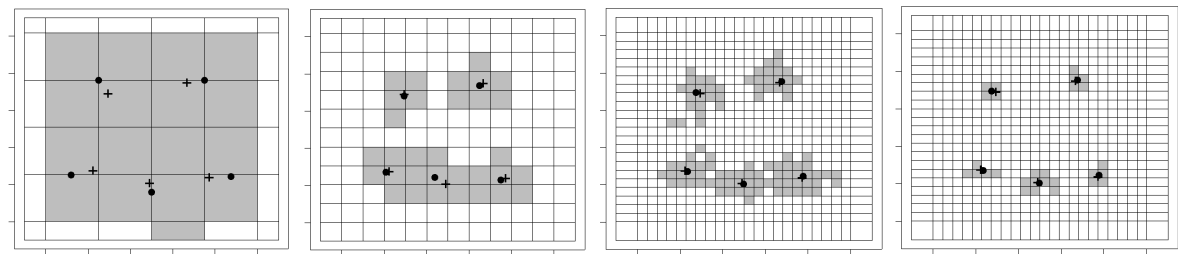

Fig. 1. Example of final definition for 2 sensors data, with 5 clusters. Each coordinate shows the actual grid for each sensor, with top- $m$ frequent states (shaded cells), gathered (circles) and real (crosses) centers, run with: $(w=6, m=20),(w=12, m=60)$, $(w=24, m=180)$, and $(w=24, m=180)$ presenting only guaranteed top- $m$.

states which is a sample of the actual states, being biased to dense cells. Moreover, although the furthest point algorithm may give guarantees on the initial centers for the clustering of the frequent states, the adaptive update is biased towards small changes in the concept generating the data streams. Each sensor $X_{i}$ produces a univariate adaptive grid. This process uses the PiD algorithm which, after the initial definition of the two layers based on $n_{i}$ examples, in $O\left(n_{i} \log p_{i}\right)$ time and $O\left(p_{i}\right)$ space, is continuously updated in constant $O\left(\log p_{i}\right)$ time and (almost) constant space. Since this is done parallel across the network, the time complexity of the discretization of one example in the entire network is $O(\log p)$ where $p=\max \left(p_{i}\right), \forall i \in\{1,2, \ldots, d\}$. The central site aggregates the state of each of the $d$ local sites. The focus is on monitoring the top- $m$ frequent global states, which are kept in $O(m d)$ space (the actual $m$ frequent states) and continuously updated in $O(m)$ time (linear search for the current state). The initial clustering of frequent states, and its subsequent adaptation is made in $O(\mathrm{~km})$ time. Data communication occurs only in one direction, between the local sites and the central site. All queries are answered by the central server. Also, this communication does not include sending the original data, rather informing the central server of the current discrete state of the univariate stream of each local site. This feature of only communicating the state when and if it has changed reduces the network's communication requirements. The main reason of this is that, in usual sensor data, sensor readings tend to be highly correlated with previously read value [15, hence tending to stay in the same discretized state. In the worst case scenario, where all $d$ local sites need to communicate their state to the central site, the system processes $d$ messages of one discrete value. However, every time the local site $X_{i}$ changes its univariate grid, the central site must be informed on the change so that it can correctly compute the points used to find the cluster centers, which imply sending $w_{i}$ values. In the worst case scenario, the central site may have to receive $O(w d)$ data, where $w=\max \left(w_{i}\right)$.

\section{Experimental Evaluation}

Evaluation of streaming algorithms is a hot-topic in research as no standard exists for evaluating models over streams of data. Hence the difficulty to define 
exact evaluation processes. Nevertheless, we conducted a series of experiments on synthetic scenarios, to assess the quality of our proposal. All scenarios were generated by applying the data generator used in [11, considering each dimension separated across sensors, in univariate streams. The global view of the network scenarios is created by mixtures of $k$ spherical Gaussians, with means $\mu_{i}$ in the unit hypercube. Each scenario was generated according to three parameters: dimensionality $d$ of the network, the number of mixture components $k$, and the standard deviation of each sensor stream in each component $\sigma$. Each scenario $(d, k, \sigma)$ is created with 100000 examples. Given the scope of this validation, the system's quality is measured by assigning each of the found cluster centers to the closest real cluster center, using a greedy strategy. The loss of the chosen assignment is given by

$$
L_{K}=\sum_{i=1}^{k} \sum_{j=1}^{d}\left(\hat{c}_{i j}-c_{i j}\right)^{2}
$$

where $\hat{c}_{i j}$ and $c_{i j}$ are the gathered and real values, respectively, for center $i$ in dimension $j$.

\subsection{Parameter Sensitivity}

For each scenario, the system's sensitivity to parameters is evaluated. Studied parameters are the granularity of the univariate adaptive grid, $w_{i}, \forall i \in\{1,2, \ldots, d\}$, and the number of frequent states to monitor, $m$. We fixed $p_{i}=1000, \forall i \in$ $\{1,2, \ldots, d\}$. We look for a good relation between the scenario ( $k$ and $d)$ and parameters $(w$ and $m)$. Our assumption is that parameters should follow $w \in O(k)$ and $m \in O\left(d k^{\beta}\right)$, for small $\beta$ (possibly $\beta=1$ ). To study this possibility, we define two factors $\omega$ and $\phi$, where $w=\omega k+1$ (allows extra cell which will mostly keep outliers) and $m=\phi w d$. As first layers in the univariate grids have size $>>20$, we set $\alpha=0.05$ stating that a first-layer cell of the univariate grid will be split if it contains more that $5 \%$ of the total number of points. The initial range is set to $[0,1]$. We set $d \in\{2,3,4,5\}$ and $\sigma=0.1$, varying $k \in\{3,4,5\}, \omega \in\{1,2,3,4,5\}$ and $\phi \in\{1,2,3,4,5\}$. Each scenario was evaluated with results averaged over 10 datasets. All $w_{i}$ are set with the same value $w=\omega k+1$. We vary $k$ within small values to inspect the ability of the system to find well-separable clusters. After aggregating all experiments, we computed Pearson's correlation between the parameters $(\omega$ and $\phi)$ and the resulting loss. The $\omega$ parameter reported (as expected) negative correlation with the loss $(\rho=-0.7524)$, as better granularity diminishes the error implied by performing clustering on a grid instead of the real values. However, higher granularity also implies higher values for $m$, so a compromise should be found to optimize computational costs. After running some empirical tests (which will be subject of thorough evaluation in the future), we found that $\omega$ should be larger than 1 , in order to allow the existence of infrequent cells between frequent ones, hence improving separability. For the $\phi$ parameter, the study reported a positive correlation with loss $(\rho=0.3832)$. Since this goes against the empirical intuition, we decided to try a different approach. Let $m=\frac{d w^{2}}{\gamma}$, with $\gamma \in\{10,8,6\}$. Tables 1 and 2 present the results gathered 
Table 1. Averaged loss over $\omega \in\{2,4\}$ and $\gamma \in\{6,8,10\}$, and 10 different data sets for each combination of parameters and scenarios, for DGCluster and Continuous K-Means

\begin{tabular}{ccccccccccc}
\hline \multicolumn{1}{c}{$k=2$} & \multicolumn{2}{c}{$k=3$} & \multicolumn{2}{c}{$k=4$} & \multicolumn{2}{c}{$k=5$} & \multicolumn{2}{c}{$k=10$} \\
$d$ & $D G C$ & $C K M$ & $D G C$ & $C K M$ & $D G C$ & $C K M$ & $D G C$ & $C K M$ & $D G C$ & $C K M$ \\
\hline $\mathbf{2}$ & 0.015305 & 0.000003 & 0.152415 & 0.000048 & 0.056467 & 0.032453 & 0.011277 & 0.000062 & $\mathbf{0 . 2 0 1 0 4 8}$ & 0.238827 \\
$\mathbf{3}$ & 0.018003 & 0.000010 & 0.181453 & 0.069749 & $\mathbf{0 . 1 0 2 5 5 7}$ & 0.157503 & 0.279721 & 0.209871 & $\mathbf{0 . 1 9 4 6 3 4}$ & 0.423646 \\
$\mathbf{4}$ & 0.006005 & 0.000012 & 0.004045 & 0.000022 & $\mathbf{0 . 1 7 8 7 8 2}$ & 0.185916 & 1.010598 & 0.436916 & 0.717055 & 0.386985 \\
$\mathbf{5}$ & 0.007152 & 0.000015 & $\mathbf{0 . 0 0 7 8 1 6}$ & 0.130807 & $\mathbf{0 . 2 0 0 1 0 0}$ & 0.256851 & $\mathbf{0 . 6 7 8 4 9 8}$ & 0.687560 & $\mathbf{0 . 5 4 4 5 3 3}$ & 0.908984 \\
$\mathbf{1 0}$ & 0.020770 & 0.000034 & $\mathbf{0 . 1 4 6 2 1 9}$ & 0.285631 & $\mathbf{0 . 3 8 8 4 1 4}$ & 0.939210 & $\mathbf{0 . 9 1 0 7 7 6}$ & 1.895545 & 2.188842 & 1.990172 \\
$\mathbf{2 0}$ & 0.094645 & 0.000065 & $\mathbf{1 . 2 0 0 4 2 0}$ & 1.386870 & $\mathbf{0 . 9 6 1 3 9 4}$ & 1.211905 & $\mathbf{1 . 6 1 0 6 2 7}$ & 2.015545 & 7.027379 & 5.083694 \\
\hline
\end{tabular}

Table 2. Averaged loss over all $k \in\{2,3,4,5,10\}$ for each fixed parameter over 10 different data sets

\begin{tabular}{ccccccc}
\hline$d$ & $\omega=2$ & $\omega=4$ & $\gamma=10$ & $\gamma=8$ & $\gamma=6$ & Continuous $K M$ \\
\hline $\mathbf{2}$ & 0.066647 & 0.107959 & 0.072887 & 0.096263 & 0.092757 & 0.054279 \\
$\mathbf{3}$ & $\mathbf{0 . 1 6 2 7 5 1}$ & $\mathbf{0 . 1 4 7 7 9 6}$ & $\mathbf{0 . 1 5 1 0 9 7}$ & $\mathbf{0 . 1 6 6 5 4 8}$ & $\mathbf{0 . 1 4 8 1 7 6}$ & 0.172156 \\
$\mathbf{4}$ & 0.377053 & 0.389541 & 0.381646 & 0.366113 & 0.402133 & 0.201970 \\
$\mathbf{5}$ & $\mathbf{0 . 2 4 2 5 3 8}$ & $\mathbf{0 . 3 3 2 7 0 1}$ & $\mathbf{0 . 2 9 5 8 6 1}$ & $\mathbf{0 . 2 8 1 1 0 9}$ & $\mathbf{0 . 2 8 5 8 8 9}$ & 0.396843 \\
$\mathbf{1 0}$ & $\mathbf{0 . 7 0 2 3 2 8}$ & $\mathbf{0 . 7 5 9 6 8 0}$ & $\mathbf{0 . 7 4 8 7 8 9}$ & $\mathbf{0 . 7 1 1 9 3 3}$ & $\mathbf{0 . 7 3 2 2 9 0}$ & 1.022118 \\
$\mathbf{2 0}$ & 2.137683 & 2.220103 & 2.188506 & 2.117922 & 2.230250 & 1.939616 \\
\hline
\end{tabular}

for different scenarios, comparing with a simple centralized online $k$-means strategy, to which we refer as Continuous $K$-Means, which is a simplification of the STREAM algorithm 22]. This strategy performs a $K$-Means at each chunk of examples, keeping only the centers gathered with the last chunk of data weighted by the amount of points that were assigned to each center. Once again we note how hard it becomes to define a clear relation between $w, d$ and $m$, although for higher values of $k$ and $d$ we could see some possible progressive paths towards an improvement in the competitiveness of our proposal. However, the identification of general parameters is always discussable. Although we plan to perform more exhaustive sensitivity tests, in order to achieve at least acceptable ranges for the parameters, we should stress that the flexibility included in the system allows for better deployment in sensor networks and resource restricted environments.

\subsection{Application to Physiological Sensor Data}

The Physiological Data Modeling Contest Workshop (PDMC) was held at the ICML 2004 and aimed at information extraction from streaming sensors data. The training data set for the competition consists of approximately 10,000 hours of this data, containing several variables: userID, sessionID, sessionTime, characteristic[1..2], annotation, gender and sensor[1..9]. We have concentrated on sensors 2 to 9 , extracted by userID, resulting in several experimental scenarios of eight sensors, one scenario per userID. For each scenario, we run the system with different values for the parameters, and compare the results both with the Continuous K-Means and full data K-Means, the latter serving as "real" centers definition. Since different sensors produce readings in different scales, we inspect the distribution of each sensor on an initial chunk of data, defining the initial ranges to percentiles $25 \%$ and $75 \%$. This process is acceptable in the sensor networks framework as expert knowledge about the range is usually available. Hence, we are also allowing the system to adapt the local grids accordingly. The system ran with $k \in\{2,3,4,5\}, \omega \in\{2,4,6,8,10\}$ and $\gamma \in\{10,8,6,4,2\}$. Also 
Table 3. Performance in terms of communicated values ( $\%$ of total examples $\times$ dimensions), evicted states ( $\%$ of total examples), cluster centers adaptations ( $\%$ of total examples) and number of guaranteed top- $m$ (\% of total $m$ ) with $k \in\{2,3,4,5\}$, $\omega \in\{2,4,6,8,10\}$ and $\gamma \in\{10,8,6,4,2\}$

\begin{tabular}{lcccc}
\hline & $k=2$ & $k=3$ & $k=4$ & $k=5$ \\
\hline Communication (\%) & $20.4 \pm 08.3$ & $26.8 \pm 09.09$ & $31.6 \pm 10.5$ & $36.0 \pm 11.4$ \\
Evicted (\%) & $18.0 \pm 12.6$ & $28.5 \pm 16.6$ & $36.5 \pm 18.5$ & $42.7 \pm 18.6$ \\
Updated (\%) & $03.2 \pm 01.6$ & $04.3 \pm 02.8$ & $05.9 \pm 04.5$ & $07.8 \pm 06.6$ \\
Guaranteed (\%) & $24.7 \pm 17.0$ & $17.4 \pm 14.1$ & $12.2 \pm 10.2$ & $10.1 \pm 09.0$ \\
Best Loss & 1329.67 & 5222.17 & 9529.40 & 34131.75 \\
Worst Loss & 114645.7 & 669330.5 & 393919.9 & 554944.9 \\
CKM Loss & 1785.97 & 112830.4 & 105091.0 & 276932.5 \\
\hline
\end{tabular}

monitored was the amount of communication and cluster adaptation in each run. Table 3 presents performance results for the physiological sensor data. Given the characteristics of sensor data, subsequent readings tend to stay in the same interval. Hence the advantage of local discretization: the system transmits only around $30 \%$ of the total amount of values, including transmissions of recalculated second layer intervals. Also, we should note that only a small part of the points require cluster center adaptation (less than 10\%). Overall we should stress that, given the flexibility of the system, a suitable combination of parameters can yield better results than centralizing the data, while preventing excessive communication in the sensor network.

\section{Conclusions and Future Work}

In this paper, we have proposed a new algorithm to perform clustering of distributed data streams, which reduces both the dimensionality and the communication burdens. Each sensor's data is processed locally, being incrementally discretized into a univariate adaptive grid, with each new value triggering a specific cell of this grid, defining the local state of the sensor. Whenever a local site change its state, that is, the triggered cell changes, the new state is communicated to a central site. This highly reduces the network's communication requirements with the central site which keeps a small list of counters of the most frequent global states. This is extremely helpful as sensor networks may include thousands of sensors, leading to an exponential number of cell combinations that should be monitored by the central site. The final clustering structure is defined and maintained by a simple partitional clustering algorithm, applied on the frequent states, which is capable of online update and adaptation to the evolution of the data. Experiments are presented in terms of sensitivity tests and application to a real-world data set. Major characteristics of the system include: compact representation of possibly infinite streams produced in distributed sensors, with possibly different granularities for each sensor; possibility to adapt discretization and central processing to resources available in the network; centralized monitoring of the global state of the network, with lightweight maintenance of 
frequent states; and fast and adaptable clustering of frequent states to estimate actual clusters centers. Current work is concentrated on determining acceptable ranges for the parameters of the system and application to more real-world data. Future work will focus on techniques to monitor the evolution of the clusters, taking advantage from the adaptive update of clusters already implemented in the system. Furthermore, we are preparing the deployment of the system in real wireless sensor networks, in order to better assess the sensitivity and advantages of the system with respect to restricted resources requirements.

Acknowledgment. The work of P.P. Rodrigues is supported by the Portuguese Foundation for Science and Technology (FCT) under the PhD Grant SFRH/BD/ 29219/2006. The authors thank FCT's Plurianual financial support attributed to LIAAD and CRACS and the participation of Project ALES II under FCT's Contract POSC/EIA/55340/2004 and Project CALLAS under FCT's Contract $\mathrm{PTDC} / \mathrm{EIA} / 71462 / 2006$.

\section{References}

1. Aggarwal, C.C., Han, J., Wang, J., Yu, P.S.: A framework for clustering evolving data streams. In: VLDB 2003, pp. 81-92. Morgan Kaufmann, San Francisco (2003)

2. Agrawal, R., Gehrke, J., Gunopulos, D., Raghavan, P.: Automatic subspace clustering of high dimensional data for data mining applications. In: Proceedings of the ACM-SIGMOD International Conference on Management of Data, Seattle, Washington, June 1998, pp. 94-105. ACM Press, New York (1998)

3. Akyildiz, I., Su, W., Sankarasubramaniam, Y., Cayirci, E.: A Survey on Sensor Networks. IEEE Communications Magazine 40(8), 102-114 (2002)

4. Bradley, P., Fayyad, U., Reina, C.: Scaling clustering algorithms to large databases. In: Proceedings of the Fourth International Conference on Knowledge Discovery and Data Mining, pp. 9-15. AAAI Press, Menlo Park (1998)

5. Charikar, M., Chen, K., Farach-Colton, M.: Finding frequent items in data streams. In: Widmayer, P., Triguero, F., Morales, R., Hennessy, M., Eidenbenz, S., Conejo, R. (eds.) ICALP 2002. LNCS, vol. 2380, pp. 693-703. Springer, Heidelberg (2002)

6. Cormode, G., Muthukrishnan, S.: What's hot and what's not: Tracking most frequent items dynamically. In: Proceedings of the 22nd Symposium on Principles of Database Systems, pp. 296-306 (2003)

7. Cormode, G., Muthukrishnan, S., Zhuang, W.: Conquering the divide: Continuous clustering of distributed data streams. In: Proceedings of the 23nd International Conference on Data Engineering (ICDE 2007), pp. 1036-1045 (2007)

8. Culler, D.E., Mulder, H.: Smart Sensors to Network the World.Scientific American (2004)

9. Datta, S., Bhaduri, K., Giannella, C., Wolff, R., Kargupta, H.: Distributed data mining in peer-to-peer networks. IEEE Internet Computing 10(4), 18-26 (2006)

10. Demaine, E.D., Lopez-Ortiz, A., Munro, J.I.: Frequency estimation of internet packet streams with limited space. In: Proceedings of the 10th Annual European Symposium on Algorithms, pp. 348-360 (2002)

11. Domingos, P., Hulten, G.: A general method for scaling up machine learning algorithms and its application to clustering. In: Proceedings of the Eighteenth International Conference on Machine Learning (ICML 2001), pp. 106-113 (2001) 
12. Ester, M., Kriegel, H.-P., Sander, J., Xu, X.: A density-based algorithm for discovering clusters in large spatial databases with noise. In: Second International Conference on Knowledge Discovery and Data Mining, Portland, Oregon, pp. 226231. AAAI Press, Menlo Park (1996)

13. Gama, J., Pinto, C.: Discretization from data streams: applications to histograms and data mining. In: Proceedings of the, ACM Symposium on Applied Computing (SAC 2006), pp. 662-667. ACM Press, New York (2006)

14. Gama, J., Rodrigues, P.P.: Data stream processing. In: Learning from Data Streams - Processing Techniques in Sensor Networks, ch. 3, pp. 25-39. Springer, Heidelberg (2007)

15. Gama, J., Rodrigues, P.P.: Stream-based electricity load forecast. In: Kok, J.N., Koronacki, J., López de Mántaras, R., Matwin, S., Mladenič, D., Skowron, A. (eds.) PKDD 2007. LNCS (LNAI), vol. 4702, pp. 446-453. Springer, Heidelberg (2007)

16. Gonzalez, T.F.: Clustering to minimize the maximum inter-cluster distance. Theoretical Computer Science 38, 293-306 (1985)

17. Guha, S., Rastogi, R., Shim, K.: CURE: An efficient clustering algorithm for large databases. In: Proceedings of the 1998 ACM-SIGMOD International Conference on Management of Data, pp. 73-84. ACM Press, New York (1998)

18. Luo, L., Abdelzaher, T., He, T., Stankovic, J.: EnviroSuite: An Environmentally Immersive Programming Framework for Sensor Networks. ACM TECS 5(3), 543$576(2006)$

19. Manku, G., Motwani, R.: Approximate frequency counts over data streams. In: Proceedings of the 28th International Conference on Very Large Data Bases, pp. 346-357 (2002)

20. Metwally, A., Agrawal, D., Abbadi, A.E.: Efficient computation of frequent and top$\mathrm{k}$ elements in data streams. In: Proceedings of the 10th International Conference on Database Theory, pp. 398-412. Springer, Heidelberg (2005)

21. Newton, R., Welsh, M.: Region Streams: Functional Macroprogramming for Sensor Networks. In: DMSN 2004 Workshop (2004)

22. O'Callaghan, L., Meyerson, A., Motwani, R., Mishra, N., Guha, S.: Streaming-data algorithms for high-quality clustering. In: Proceedings of the Eighteenth Annual IEEE International Conference on Data Engineering, pp. 685-696. IEEE Computer Society Press, Los Alamitos (2002)

23. Subramaniam, S., Palpanas, T., Papadopoulos, D., Kalogeraki, V., Gunopulos, D.: Online outlier detection in sensor data using non-parametric models. In: VLDB, pp. 187-198 (2006)

24. Wang, W., Yang, J., Muntz, R.R.: STING: A statistical information grid approach to spatial data mining. In: Proceedings of the Twenty-Third International Conference on Very Large Data Bases, Athens, Greece, pp. 186-195. Morgan Kaufmann, San Francisco (1997)

25. Zhang, T., Ramakrishnan, R., Livny, M.: BIRCH: An efficient data clustering method for very large databases. In: Proceedings of the 1996 ACM SIGMOD International Conference on Management of Data, pp. 103-114. ACM Press, New York (1996) 